Full Length Article

\title{
Microglia depletion prior to lipopolysaccharide and paraquat treatment differentially modulates behavioral and neuronal outcomes in wild type and G2019S LRRK2 knock-in mice
}

\author{
Zach Dwyer $^{\mathrm{a}, 1}$, Chris Rudyk ${ }^{\mathrm{a}}$, Divya Situt ${ }^{\mathrm{a}}$, Sheryl Beauchamp ${ }^{\mathrm{a}}$, Jawaria Abdali ${ }^{\mathrm{a}}$, Anu Dinesh ${ }^{\mathrm{a}}$, \\ Nathalie Legancher $^{\mathrm{b}}$, Hongyu Sun ${ }^{\mathrm{a}}$, Michael Schlossmacher ${ }^{\mathrm{b}, \mathrm{c}, 1}$, Shawn Hayley ${ }^{\mathrm{a}, *, 1}$, CLINT \\ (Canadian LRRK2 in inflammation team) \\ a Department of Neuroscience, Carleton University, 1125 Colonel By Drive, Ottawa, Ontario, K1S 5B6, Canada \\ ${ }^{\mathrm{b}}$ University of Ottawa, Canada \\ ${ }^{\mathrm{c}}$ Ottawa Hospital Research Institute, Canada
}

\section{A R T I C L E I N F O}

\section{Keywords:}

Microglia

Neurodegeneration

Parkinson's

LRRK2

LPS

Toxicant

Cytokine

Stress

\begin{abstract}
A B S T R A C T
Background: Substantial data have implicated microglial-driven neuroinflammation in Parkinson's disease (PD) and environmental toxicants have been long expected as triggers of such inflammatory processes. Of course, these environmental insults act in the context of genetic vulnerability factors and in this regard, leucine rich repeat kinase 2 (LRRK2), may play a prominent role.

Methods: We used a double hit, lipopolysaccharide (LPS; endotoxin) followed by paraquat (pesticide toxicant) model of PD in mice with the most common LRRK2 mutation G2019S, knockin mice and wild type littermates. In order to assess the contribution of microglia, we depleted these cells (through 14 days of the CSF-1 antagonist, PLX-3397) prior to LPS and paraquat exposure.

Results: We found that the G2019S mice displayed the greatest signs of behavioral pathology, but that the PLX3397 induced microglial depletion at the time of LPS exposure diminished toxicity and weight loss and blunted the reduction in home-cage activity with subsequent paraquat exposure. However, neither the PLX-3397 pretreatment nor the G2019S mutation affected the LPS + paraquat induced loss of substantia nigra pars compacta (SNc) dopamine neurons or elevation of circulating immune (IL-6) or stress (corticosterone) factors. Intriguingly, microglial morphological ratings were basally enhanced in G2019S mice and the PLX-3397 pre-treatment reversed this effect. Moreover, PLX-3397 pre-treatment selectively elevated soluble a-synuclein and SIRT3 levels, while reducing SNc caspase-1 and 3, along with CX3CR1. Hence, the re-populated "new" microglia following cessation of PLX-3397 clearly had an altered phenotype or were immature at the time of sacrifice (i.e. after 11 days).

Conclusions: Collectively, these findings suggest that G2019S knock-in and PLX-3397 microglial depletion at the time of LPS exposure affects behavioral, but not neurodegenerative responses to subsequent environmental toxin exposure.
\end{abstract}

\section{Introduction}

Parkinson's disease (PD) is a progressive neurodegenerative disease in which the main risk factor is age, with epidemiological and experimental studies also implicating environmental and inflammatory insults as potential triggers (Uversky et al., 2001; Baltazar et al., 2014; Dick et al., 2007). Similar to the peripheral immune system, the brain's specialized immunocompetent cell, microglia, have surveillance processes to detect and eliminate pathogens or other threats within their microenvironment (Neumann et al., 2009). However, there is now substantial evidence demonstrating that microglia driven inflammation when chronic and excessive can also contribute to the progression of PD

\footnotetext{
* Corresponding author. 1125 Colonel By Drive, Ottawa, Ontario, K1S 5B6, Canada.

E-mail address: shawn_hayley@carleton.ca (S. Hayley).

1 Membership of the Canadian Lrrk2 in Inflammation Team (CLINT) is listed in the Acknowledgments.
} 
(German et al., 2011). Accordingly, augmented microglial reactivity and levels of pro-inflammatory cytokines have been observed PD patients (German et al., 2011; Sawada et al., 2006; Litteljohn et al., 2010) and systemic infections may exaggerate behavioral changes (Ascherio and Schwarzschild, 2016; Wu et al., 2015). We and others have demonstrated that exposure to the bacterial endotoxin, lipopolysaccharide (LPS), augmented dopamine neuron loss in mice that were subsequently exposed to the pesticide paraquat (which has been linked to PD as a relevant environmental toxicant) and that this effect was related to microglial hyperactivity (Purisai et al., 2007; Mangano and Hayley, 2009).

Of course, environmental insults act within the context of genetic vulnerabilities and in this regard the most commonly mutated gene in both sporadic and familial PD is Leucine-rich-repeat kinase 2 (LRRK2). Specifically, the Gly2019Ser (G2019S) mutation is most common and is approximately $30 \%$ penetrant at the age of 70 , indicating that further environmental factors may modulate its impact (Lee et al., 2017a; Marder et al., 2015). The G2019S mutation causes an approximate 3-fold increase in kinase activity and its main identified interactors are Rabs and WAVE2, both proteins being heavily involved in cell motility and phagocytosis (Kim et al., 2018; Liu et al., 2018; Moehle et al., 2015). Further, recent studies demonstrated that the highest levels of LRRK2 is in immune cells, including circulating B lymphocytes, dendritic cells and macrophages (Hakimi et al., 2011; Cook et al., 2017a). LRRK2 was also recently shown to regulate macrophage and microglial motility and phagocytosis is a target gene induced by interferon-gamma (Kuss et al., 2014), which we previously implicated in the inflammatory and neurodegenerative response elicited by either MPTP or paraquat treatment (Mangano et al., 2012; Mount et al., 2007). Thus, LRRK2 could be critical for the inflammatory responses to PD relevant toxins, such as paraquat, as well as typical bacterial insults, as mimicked by LPS.

Given the postulated role of microglia in PD, it was of interest to assess how transient microglial depletion at the time of LPS and paraquat exposure impacts behavioral and neuronal outcomes and whether the G2019S mutation differentially affects these outcomes. Accordingly, pharmacological inhibition of the colony stimulating factor-1 (CSF-1) receptor using the drug, PLX-3397 has recently proven effective in depleting microglia. Indeed, its daily administration ( $>7$ days) leads to a $>90 \%$ depletion of microglia and macrophages which appears to mostly recover within 1-2 weeks following PLX-3397 cessation (Elmore et al., 2014). We presently found that PLX-3397 induced depletion of microglia blunted the impact of intra-SNc LPS infusion and subsequent paraquat exposure on behavioral outcomes, but had no effect on the loss dopamine neurons or elevation of cytokine or corticosterone levels that was induced by LPS and paraquat combination treatment. The phenotype of the re-populated microglia following PLX-3397 cessation may have been altered, since levels of soluble a-synuclein, SIRT3, CX3CR1 and caspases 1 and 3 were all changed in mice previously treated with the drug. Finally, the G2019S knockin mutation augmented behavioral toxicity and appeared to have basally elevated changes in microglial morphology but had little influence on other outcomes. In short, we provide evidence that the G2019S mutation might augment toxicity of LPS and paraquat and that transient microglial depletion can blunt certain aspects of pathology but not the basic neurodegenerative process.

\section{Methods}

\subsection{Animals}

96 male LRRK2 G2019S knock-in mice (Herzig et al., 2011) (Novartis; a kind gift from the lab of Dr. David S. Park) and WT littermates were generated via heterozygote-heterozygote pairings. All animals were group housed in individually ventilated cages on a $12 \mathrm{H}$ light cycle and received ad libitum access to tap water and either AIN-76 or AIN-76 with incorporated PLX-3397 rodent chow. Until 3 months of age, animals additionally had access to environmental enrichment (red nicram houses and nestlets). They were then individually housed for one week with only a nestlet after which time they entered into the experimental conditions.

\subsection{PLX-3397 treatment}

Mice in the AIN-76 control group received AIN-76 ad libitum for the course of the experiment (See Fig. 1). Mice in the PLX-3397 treatment groups received PLX-3397 incorporated in AIN-76 rodent chow from Research Diets Inc. (New Brunswick, NJ, USA) as previously reported (Elmore et al., 2014).

\subsection{Stereotaxic surgery: LPS infusion}

At three months of age, all animals underwent stereotaxic surgery. Following $5 \%$ isoflourane anesthetic and $20 \mathrm{mg} / \mathrm{kg}$ of the analgesic tramadol, mice were secured in a Kopf Instruments Model 940 stereotaxic frame and a small, $1 \mathrm{~cm}$, incision was made in the centre of the skull and a $1.5 \mathrm{~mm}$ diameter hole was drilled in the skull at $\mathrm{x}=1.2 \mathrm{~mm}$ and $\mathrm{y}$ $=3.14 \mathrm{~mm}$ relative to Bregma over the left hemisphere. A 22-gauge injector was used to infuse half of each group with $2 \mu \mathrm{L}$ of either saline or $1 \mu \mathrm{g} / \mu \mathrm{L}$ LPS directly above the SNc (at $4 \mathrm{~mm}$ below the surface of the skull). A Harvard Apparatus picopump was used to ensure a constant infusion over $4 \mathrm{~min}$. The injector was left in place for $5 \mathrm{~min}$ after the infusion to allow the LPS/saline to absorb into the tissue before slowly being removed. The hole in the skull was filled with Bone Wax ${ }^{\circledR}$ before suturing. The animals then received $100 \%$ oxygen for $15 \mathrm{~s}$ and were placed into recovery cages situated half-on half-off a $37{ }^{\circ} \mathrm{C}$ circulating water heating pad. Animals were given hydrogel for 4 days after surgery and $20 \mathrm{mg} / \mathrm{kg}$ tramadol subcutaneously twice a day for 3 days following surgery.

\subsection{Paraquat injections}

At two days following LPS/saline infusion, animals who received intra-SNc LPS also began receiving i.p. paraquat injections, whereas animals infused with saline received further i.p. saline injections. Hence, each animal received either $10 \mathrm{mg} / \mathrm{kg}$ of paraquat or an equivalent volume of saline. Paraquat was freshly made each morning and these injections were given every $48 \mathrm{~h}$ for 11 days for a total six injections.

\subsection{Behavioral analyses}

Animals were weighed each morning prior to PLX-3397 administration as well as twice daily for the first 4 days after surgery and finally in the morning of each paraquat/saline injection until sacrifice.

\subsection{Home-cage locomotor activity}

Spontaneous home cage locomotor activity was measured over a complete $12 \mathrm{~h}$ light/dark cycle using a Micromax (MMx) infrared beambreak apparatus (Accuscan Instruments, Columbus, OH, USA), as previously described (Litteljohn et al., 2014). Spontaneous home cage locomotor activity assessment was completed following a 30-min acclimation period in our behavioural testing room post nestlet removal, measurements of home-cage locomotor activity occurred on the nights of days 1 , 13, 21 and 25 of the experiment.

\subsection{Brain dissection and tissue extraction}

Half the animals were intraperitoneally administered $200 \mathrm{mg} / \mathrm{kg}$ of sodium pentobarbital and perfused with $4 \%$ paraformaldehyde. Twentyfour hours later the brains were transferred to $10 \%$ sucrose and then transferred to $30 \%$ sucrose $48 \mathrm{~h}$ after sacrifice. The remaining animals were sacrificed via rapid decapitation and trunk blood, brains and organs were extracted and flash frozen at $-80^{\circ} \mathrm{C}$.

Following rapid decapitation, brains were excised and sectioned into 


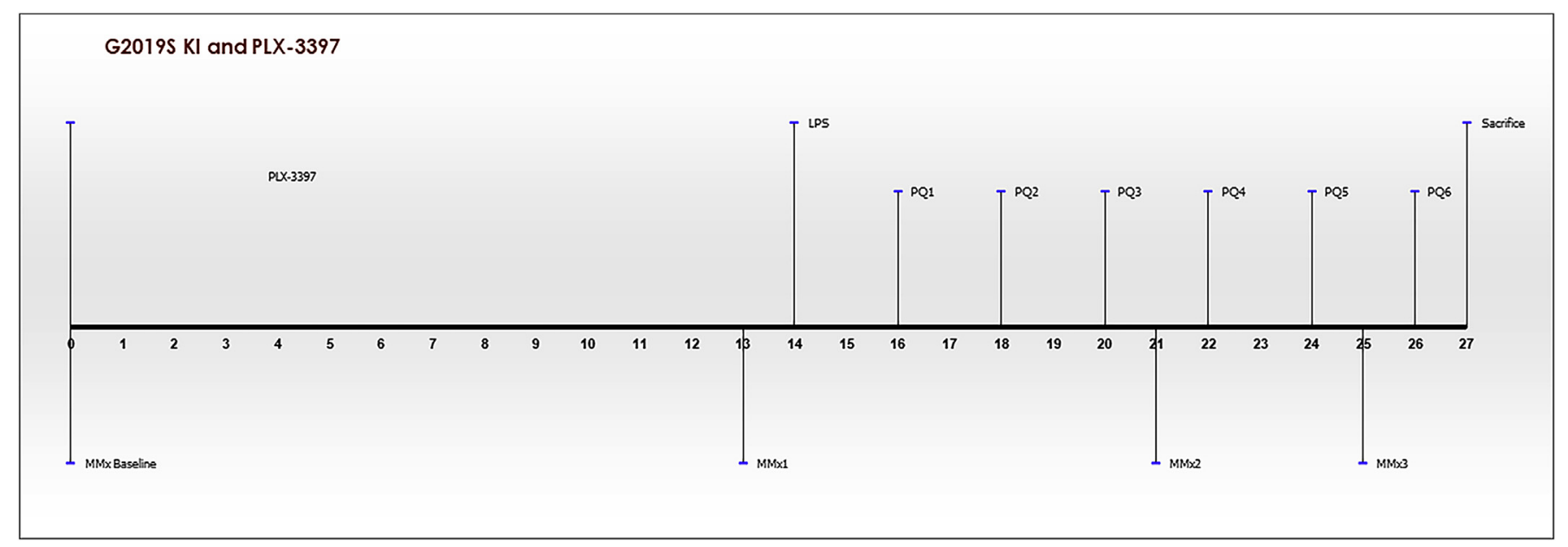

Fig. 1. Timeline with injection days and treatments indicated by vertical bars above the axis while behavioural assessments are indicated below the axis.

sequential coronal slices using razor blades and a chilled stainless-steel microdissection matrix with adjacent slots spaced $\sim 0.5 \mathrm{~mm}$ apart. Hollow biopsy needles were then used to collect the dorsal striatum (ST) and SNc. The tissue was immediately frozen upon dissection and was stored at $-80{ }^{\circ} \mathrm{C}$ until processing.

\subsection{Plasma corticosterone assay}

At the time of decapitation, trunk blood from all of the animals, was collected in tubes containing $10 \mu \mathrm{g}$ EDTA. Samples were centrifuged ( $2000 \mathrm{~g}$ for $20 \mathrm{~min}$ ) and the plasma removed and stored in aliquots at $-80{ }^{\circ} \mathrm{C}$ for later corticosterone determination with commercially available ELISA kits (Enzo, NY, USA \#ADI-900-097). Samples were assayed in duplicate within a single run to control for inter-assay variability; the intra-assay variability was less than $10 \%$.

\subsection{Plasma determination of cytokines}

Trunk blood was collected at time of decapitation and prepared as for the corticosterone assay in a separate aliquot at $-80^{\circ} \mathrm{C}$. IL- $1 \mathrm{~b}$, IL-6, IL-10 and TNF-a levels were determined using a Luminex Immunoassay (R\&D Systems, NE, USA) run following kit instructions on a Luminex Magpix (Luminex Corporation, TX, USA). Samples were assayed in duplicate within a single run to control for inter-assay variability; the intra-assay variability was less than $10 \%$.

\subsection{Alpha synuclein ELISA}

The amount and classification of alpha-synuclein in the posterior striatum was assessed through ELISA following serial protein extraction. Briefly, samples were suspended in TSS buffer (140 mM NaCl, 5 mM TrisHCL, in water) with a Roche EDTA-free proteinase inhibitor. Samples were then homogenized on ice using 50 strokes of a Wheaton tissue deuce. Homogenates were placed in a $4{ }^{\circ} \mathrm{C}$ chilled micro ultracentrifuge (Sorvall, Waltham MA) and spun for $30 \mathrm{~min}$ at 130,000 RCF. The supernatant was then removed which constituted the soluble fraction, and the pellet was suspended in TSS buffer with $1 \%$ SDS. The suspension was allowed to sit at room temperature for $10 \mathrm{~min}$ then centrifuged at $10^{\circ} \mathrm{C}$ and $130,000 \mathrm{RCF}$ for a further $30 \mathrm{~min}$. The supernatant was again removed as the insoluble fraction and following BCA quantification samples were frozen at $-80^{\circ} \mathrm{C}$ until processed. These samples were then analyzed using a sandwich ELISA as previously described (Tomlinson et al., 2017).

\subsection{Immunohistochemistry}

In order to examine microglial reactivity sections were stained with ionized calcium-binding adapter molecule 1 (IBA1) and to asses dopamine cell survival, we used tyrosine hydroxylase (TH). Brains were sliced into $40 \mu \mathrm{m}$ thick sections on our Shandon AS620 cryostat (Fisher Scientific) and immediately placed in a $0.1 \mathrm{M}$ PB solution containing $0.1 \%$ sodium azide ( $\mathrm{pH} 7.4)$. Every third section was selected for each stain (i.e. ST TH; SNc TH; SNc IBA1). For SNc TH staining, slices were washed in phosphate buffer saline (PBS) (pH 7.4) three times for 5 min each, followed by a 30 -min incubation in $0.3 \%$ hydrogen peroxide in PBS. Sections were then washed in PBS three times 5 min each and a 1-h incubation in blocking solution containing $5 \%$ normal goat serum (NGS), $0.3 \%$ triton-X, with $0.1 \mathrm{M}$ PBS (pH 7.2). Blocker was removed and the slices were then incubated overnight in primary antibody solution $(5 \%$ NGS, $0.3 \%$ Triton- $\mathrm{X}, 0.3 \%$ bovine serum albumin (BSA) in $0.1 \mathrm{M}$ PBS) with 1:2000 anti-mouse TH (Immunostar, Hudson, WI). The following day the primary antibody solution was removed and sections were washed in PBS three times for a period of $5 \mathrm{~min}$ each. Following the washes, antibodies in secondary solution (1.6\% NGS, $0.16 \%$ Triton X, $0.3 \%$ BSA, in $0.1 \mathrm{M}$ PBS) were applied to striatum (anti-mouse IgG; $1: 500$ ) for a period of $2 \mathrm{~h}$, and to SNc (anti-mouse HRP; 1:200) sections for $4 \mathrm{~h}$. Following this, three times 5-min washes were applied to the striatum which was then incubated again in secondary solution with HRP (1:1000) for an additional $2 \mathrm{~h}$. All sections were given three times $5 \mathrm{~min}$ PBS washes and sequentially exposed to a DAB reaction containing Tris$\mathrm{HCl}, \mathrm{DAB}$ and $0.6 \%$ hydrogen peroxide in $\mathrm{dH}_{2} \mathrm{O}$ for visualization. Sections were washed in PBS three times 5 min each following DAB exposure and all sections were then slide mounted and set to dry overnight. Sections were dehydrated using a series of alcohol and clearene washes and subsequently cover-slipped using DPX. All incubations occurred at room temperature.

To label IBA1, processed ST and SNc slices were washed in PBS $(\mathrm{pH}$ 7.2) three times for $5 \mathrm{~min}$ each, followed by a 1-h incubation in blocking solution ( $5 \%$ NGS, $0.3 \%$ triton-X, in $0.1 \mathrm{M}$ PBS). Following removal of the blocker, slices were then placed in anti-rabbit IBA1 (Abcam, Cambridge, MA) at a dilution of 1:1000 in primary solution ( $5 \%$ NGS, $0.3 \%$ Triton-X, $0.3 \% \mathrm{BSA}$ in $0.1 \mathrm{M} \mathrm{PBS}$ ) for a period of $2 \mathrm{~h}$. Sections were then washed in $0.1 \mathrm{M}$ PBS three times for a period of $5 \mathrm{~min}$ each and reacted with either 1:1000 of anti-goat Alexafluor 594 or 647 antibody in primary solution ( $5 \%$ NGS, $0.3 \%$ Triton-X, $0.3 \%$ BSA in 0.1 M PBS). The signal was visualized with immunofluorescence microscopy using Microbrightfield image acquisition software on a Zeiss Axioimager2 microscope. All slices were selected and compared between animals at the same distance from bregma.

$\mathrm{DAB}$ stained $\mathrm{TH}+$ cells were analyzed using the optical fractionator 
workflow in Stereoinvestigator (MBF, Williston, VT, USA) as previously described (Mangano et al., 2011). A total of six slices between bregma levels -3.08 and -3.52 were counted by a blind observer under a $63 \mathrm{X}$ oil immersion lens.

\subsection{Western blot}

Brain tissue punches were collected to detect levels of SIRT 3, Caspase 1 and 3, CX3CR1, and GFAP as described previously (Rudyk et al., 2019). Briefly, whole cell lysates were homogenized in Radio Immuno Precipitation Assay (RIPA) buffer [50 mM Tris (pH 8.0), $150 \mathrm{mM}$ sodium chloride, $0.1 \%$ sodium dodecyl sulphate (SDS), $0.5 \%$ sodium deoxycholate and 1\% Triton X-100] mixed with 1 tablet of Complete Mini ethylenediaminetetraacetic acid (EDTA)-free protease inhibitor (Roche Diagnostics, Laval, QC, Cat \#11 836170 001) per $10 \mathrm{~mL}$ of buffer. On the first day of analysis, proteins were separated using sodium dodecyl sulphate-polyacrylamide gel electrophoresis (SDS-PAGE). In order to determine total protein, membranes were incubated in REVERT total protein solution for a period of $5 \mathrm{~min}$ followed by placement into a
REVERT wash solution (6.7\% Glacial Acetic Acid, 30\% Methanol, in water) two times 2 min each. Membranes were then quickly rinsed with distilled water and imaged on our LI-COR Odyssey imaging system on the 700 channel for an exposure period of $2 \mathrm{~min}$. Membrane incubation with rabbit anti- SIRT 3 (1:1000), Caspase 1 and 3 (1:1000), CX3CR1 (1:2000), and GFAP (1:4000) for a period of $60 \mathrm{~min}$ in $0.05 \%$ fish gelatin in TBS with $0.1 \%$ tween followed by $1 \mathrm{~h}$ in infrared anti-rabbit conjugate at a concentration of $1: 20000$ in $0.5 \%$ fish gelatin solution containing $0.2 \%$ tween and $0.01 \%$ SDS. Any unbound antibody was removed using $15 \mathrm{~mL}$ of TBS-T/membrane and membranes washed and read on our Licor Odyssy system at the appropriate wavelength for $6 \mathrm{~min}$.

\subsection{Statistical analysis}

All data was analyzed by 2 (Genotype; WT vs. KI) X 2 (Diet; AIN-76 vs. PLX-3397) X 2 (Toxin: Vehicle vs LPS-paraquat) ANOVA with significant interactions further analyzed by means Bonferroni follow up comparisons $(\mathrm{p}<0.05)$ where appropriate. Data is presented in the form of mean \pm standard error mean (mean \pm SEM). All data was analyzed using the

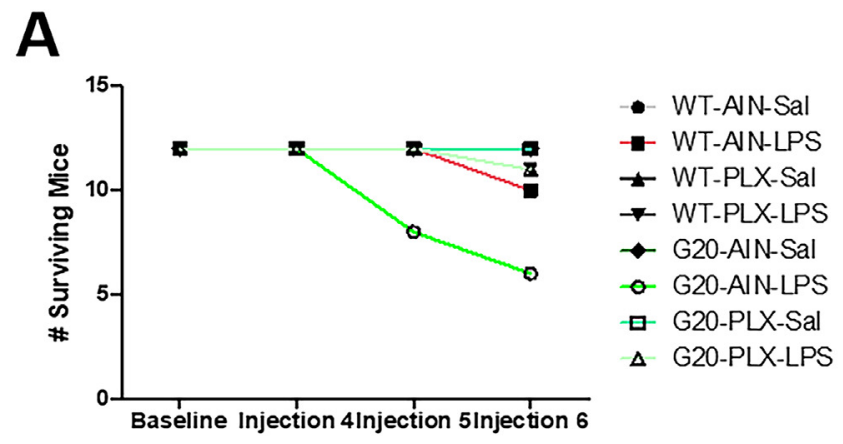

B 48 Hours Post LPS Weight
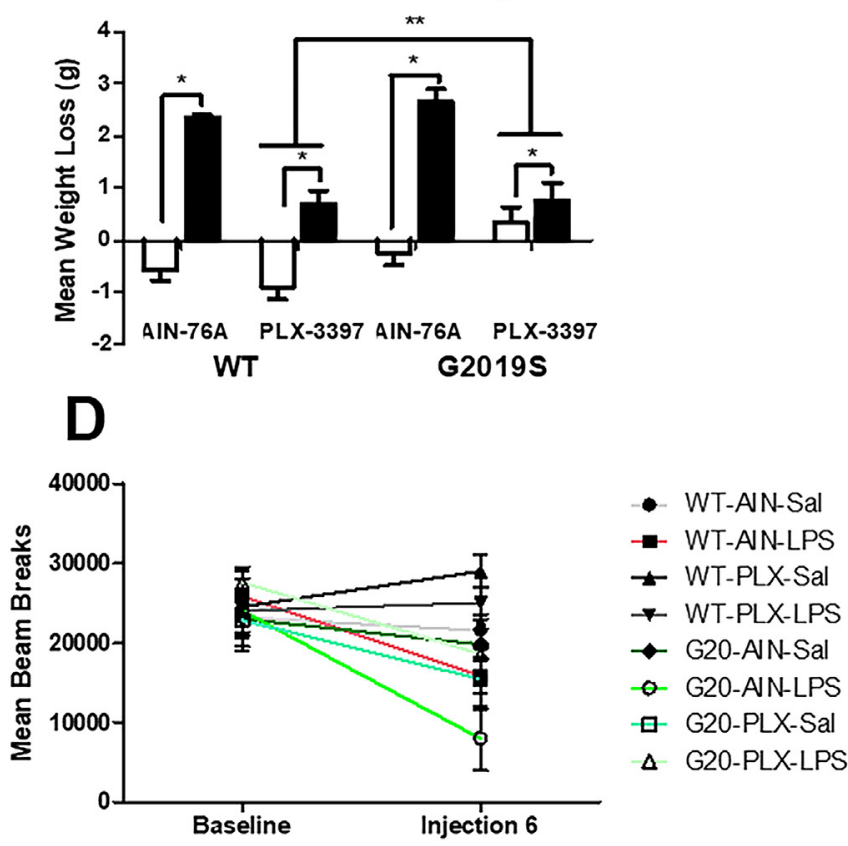

C Weight at Final Paraquat Injection
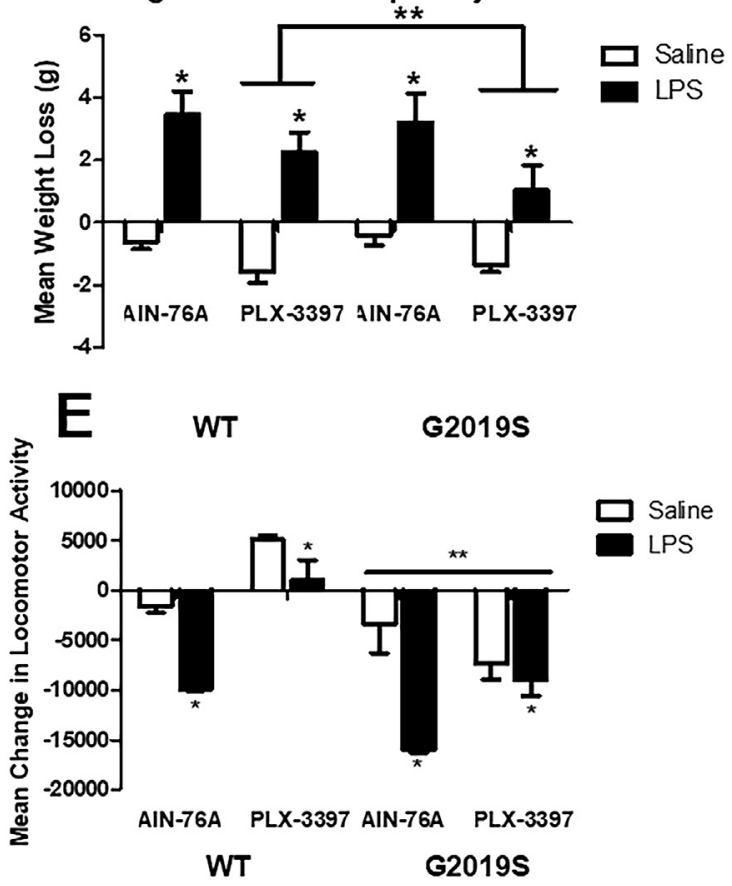

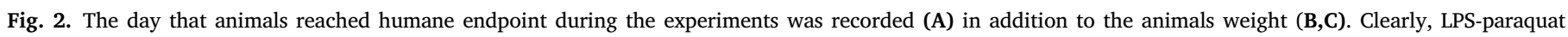

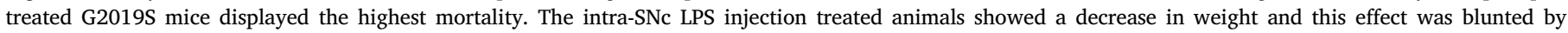

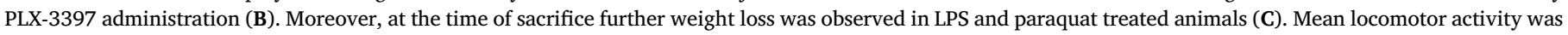

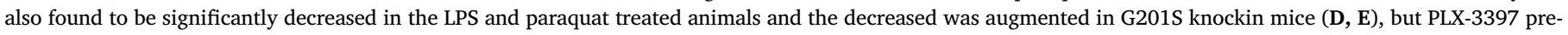
treatment blunted this response (D,E). ${ }^{*} \mathrm{p}<0.05$ compared to control groups, ${ }^{* *} \mathrm{p}<0.01$ compared to control groups. 
statistical software StatView (version 6.0) and differences were considered statistically significant when $\mathrm{p}<0.05$.

\section{Results}

\subsection{PLX-3397 pre-treatment protected mice from LPS induced weight loss and locomotor deficits}

LRRK2 G2019S KI mice showed significant mortality when treated with LPS and paraquat, but this effect was prevented in animals pretreated with PLX-3397 (Fig. 2A). Central LPS injection induced weight loss in mice regardless of genotype; however, treatment with PLX-3397 again ameliorated this effect $(F(1,84)=19.145 p<0.001)$ (Fig. 2B). Following LPS and paraquat treatment, just prior to sacrifice, significant weight loss was still observed, irrespective of genotype, but this was again blunted in mice previously treated with PLX-3397 (Treatment: $F(1,90)=67.222, p<0.001$, Diet: $F(1,90)=9.448, p<0.01$ ) (Fig. $2 \mathrm{C}$ ). Mean home-cage locomotor activity was significantly decreased by the LPS and paraquat treatment and this effect was especially pronounced in the G2019S KI mice. Once again, pre-treatment with PLX-3397 totally prevented this effect in WT mice and also blunted it in the G2019S mutants $(F(1,54)=5.040, p<0.05)$ (Fig. 2D,E).

3.2. LPS and paraquat combination treatment leads to $\mathrm{TH}+$ neuronal loss in the SNc in both WT and G2019S KI mice

Stereological counts of TH positive SNc cells revealed an obvious statistically significant loss of neurons in mice that received the LPS plus paraquat treatment $(F(1,24)=30.833, p<0.001)$, Fig. 3). In particular, the LPS and paraquat treatment resulted in an approximate $30-40 \%$ lesion in WT and 15-20\% in the G2019S mutants, which in both cases were significantly less than their respective saline treated mice ( $\mathrm{p}<$ 0.05). That said, there was no significant effect of genotype and similarly, the number of surviving TH + neurons were unaffected by the PLX-3397 treatment (Fig. 3).

3.3. LPS and paraquat treatment increased microglial activation, as does the LRRK2 G2019S genotype and this treatment effect is reduced by PLX3397 administration

Microglial morphological state (as an index of activation) was assessed by immunofluorescent IBA-1 staining by a blind rater using a validated microglial rating scale (Bobyn et al., 2012). As expected, there was a significant main effect, such that LPS and paraquat exposure significantly increased microglial morphology ratings $(F(1,35)=15.692$, $p<0.001$ ) (Fig. 4). Most strikingly however, there also was a significant main effect for genotype, such that the LRRK2 G2019S mutant mice had very marked microglial changes that was evident even in the absence of the toxin treatments $(F(1,35)=12.320, p=0.001$ ) (Fig. 4). Finally, pre-treatment with PLX-3397 did significantly blunt the microglial ratings, however, this effect was admittedly modest $(F(1,35)=4.527, p<$ 0.05) (Fig. 4).

\subsection{LPS and paraquat combination treatment increases circulating corticosterone and cytokines}

All mice treated with LPS and paraquat, irrespective of genotype or PLX 3397 treatment, displayed a similar magnitude and statistically significant rise in circulating corticosterone $(F(1,38)=12.775, p<$ 0.001 ) (Fig. 5A). Animals treated with LPS and paraquat were also found
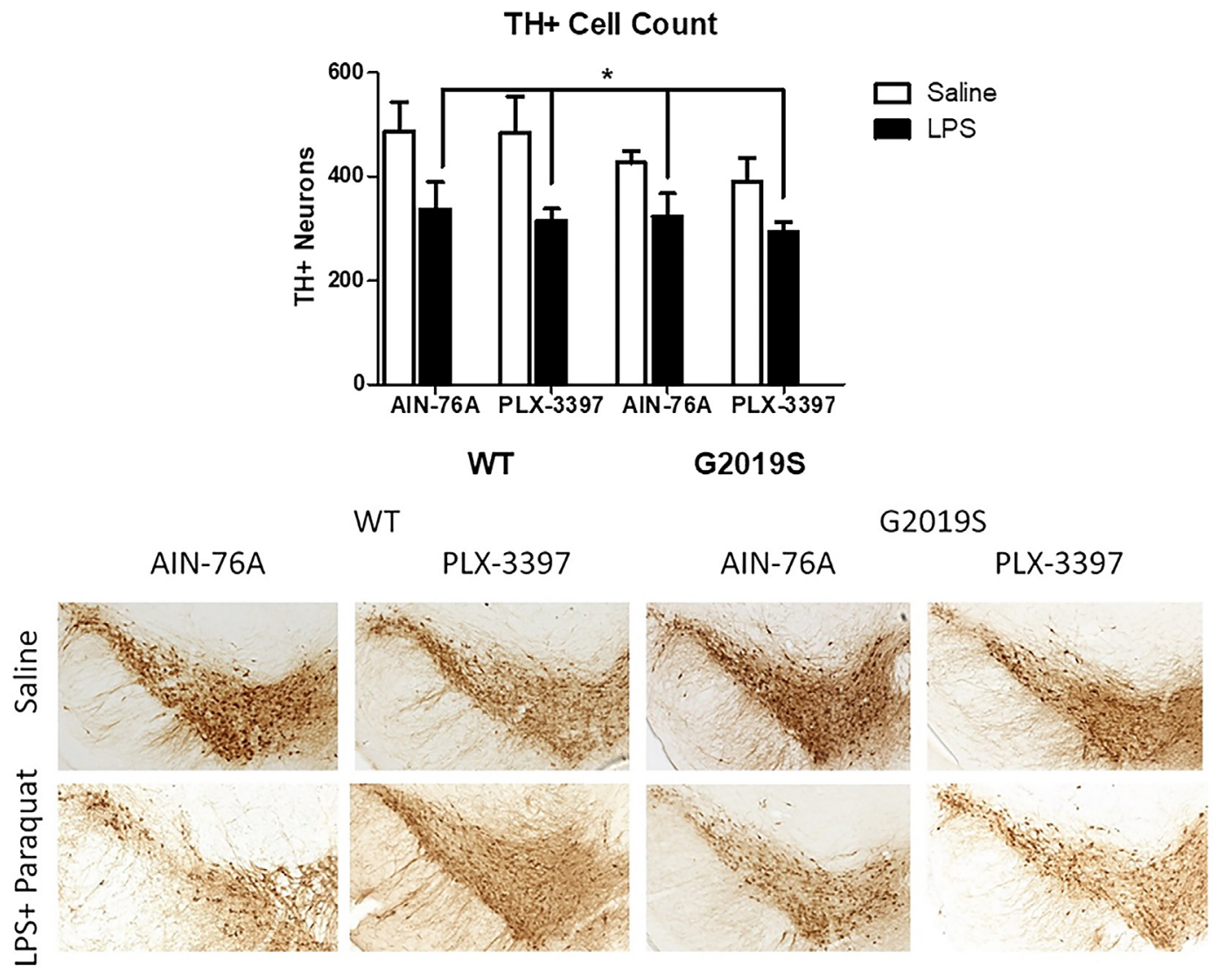

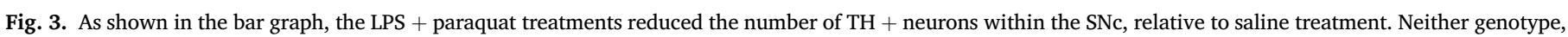

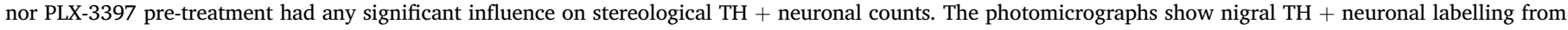
representative brain sections. ${ }^{*} \mathrm{p}<0.05$, relative to saline treated mice. 


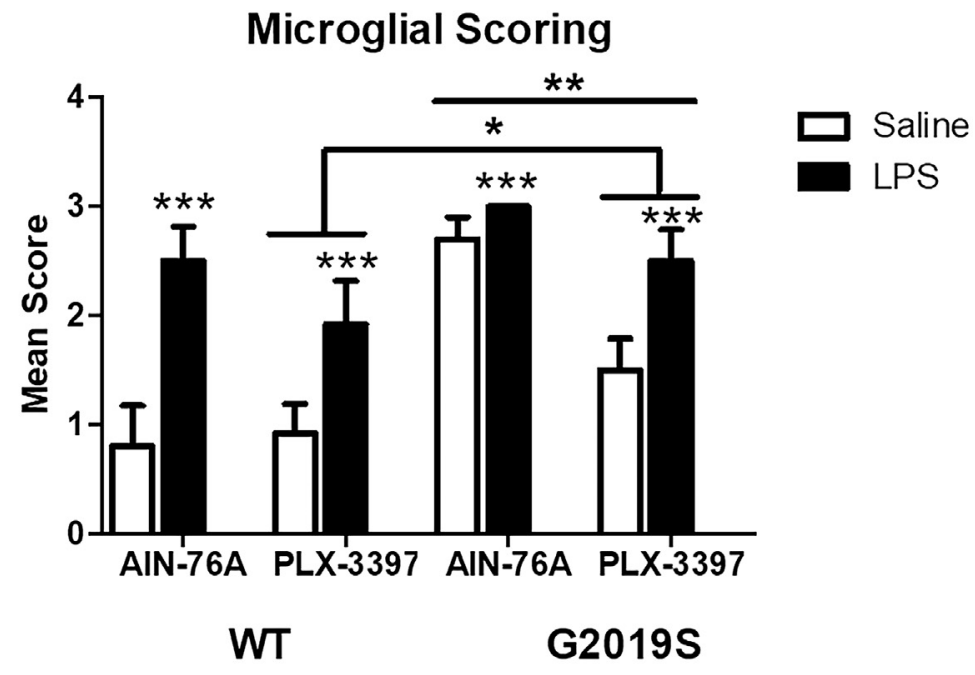

WT

AIN-76A
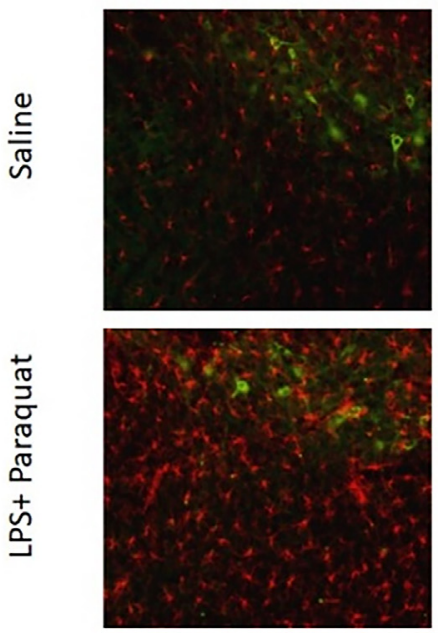

PLX-3397
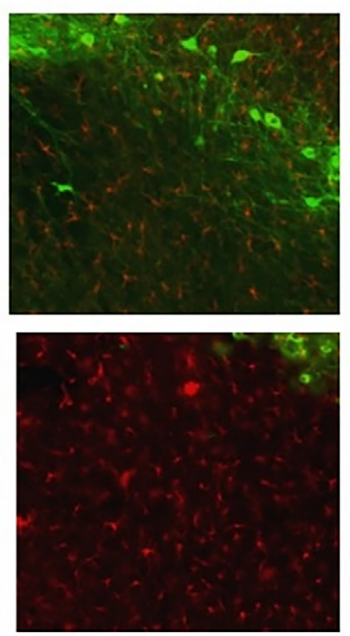

G2019S
G2019S

AIN-76A
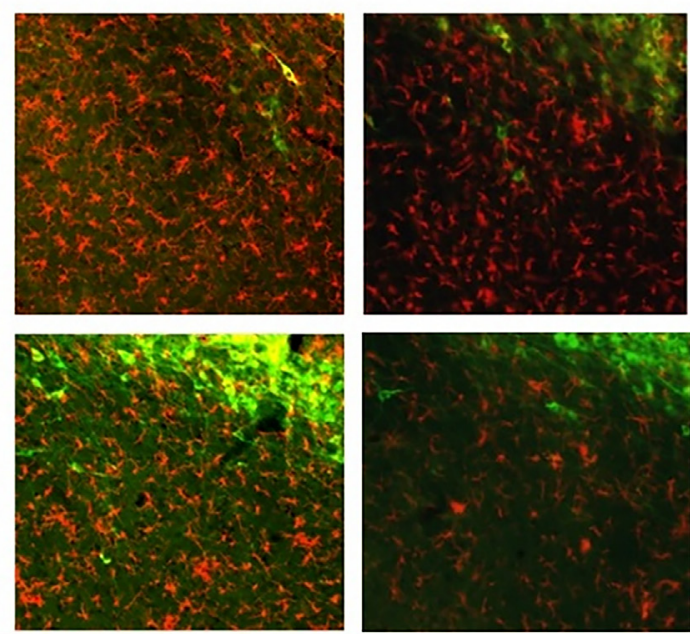

Fig. 4. As depicted in the bar graph (top) and representative photomicrographs (bottom), morphological analyses revealed marked changes in microglial ratings in the SNc between the groups. The LPS and paraquat combination treatment markedly increased ratings of microglial morphology. As well, the G2019S mutation was found to significantly promote microglial morphology changes; however, this effect was blunted in mice that were previously treated with PLX-3397. *p $<0.05$, relative to saline treated mice.

to have elevated levels of the pro-inflammatory cytokine, IL-6 $(F(1,39)=$ 4.793, $p<0.05$ ) (Fig. 5B) and again, there was no effect of either genotype or PLX 3397. In contrast, the anti-inflammatory cytokine, IL-10, was unaffected by LPS and paraquat but was significantly elevated in the G2019S mutant mice $(F(1,39)=8.066, p<0.01)$ (Fig. 5 C). No statistically significant differences were detected in circulating TNF- $\alpha$; however, a modest non-significant rise was detected in the LPS and paraquat treated groups $(F(1,39)=2.877, p=0.09$ ) (Fig. 5D).

3.5. PLX-3397 administration decreased levels of cell death markers and the fraktalkine receptor while increasing levels of the anti-oxidant inducer SiRT3

Levels of the fractalkine receptor, CX3CR1, caspase-1 and caspase- 3 were all robustly and significantly reduced by pre-treatment with PLX3397 (Fig. 6 A $F(1,33)=23.179 p<0.001$, C $F(1,33)=36.230, p<$ 0.001 , and $\mathrm{D} F(1,40)=11.505, p<0.01)$. In contrast, levels of the mitochondrial anti-oxidant inducer, SiRT3, were significantly increased by the PLX-3397 treatment $(F(1,40)=23.187, p<0.001$ ) (Fig. 6 B). Intriguingly, the LPS and paraquat treatment did not influence any of these factors and nor did genotype.

3.6. Markers of astrogliosis are increased following LPS and paraquat administration while pre-treatment with PLX-3397 led to further GFAP increases

The astrocytic marker, GFAP, was significantly elevated within the SNc by the LPS-paraquat treatment $(F(1,33)=10.196, p<0.01$ ) (Fig. 7). Moreover, this effect was greater still in the G2019S mutant mice Curiously, pre-treatment with the CSF-1 inhibitor, PLX-3397, also resulted in increased in nigral GFAP levels and this was selectively observed in the G2019S mutants $(F(1,33)=9.228, p<0.01)$ (Fig. 7).

\subsection{PLX-3397 administration leads to increased soluble alpha synuclein in the striatum}

Alpha synuclein levels were evaluated by both western blot and ELISA in order to determine alterations in total and serially extracted protein in the striatum. Total alpha-synuclein as assessed by western blot on RIPAbuffer extracted striatum samples showed significantly elevated striatal 

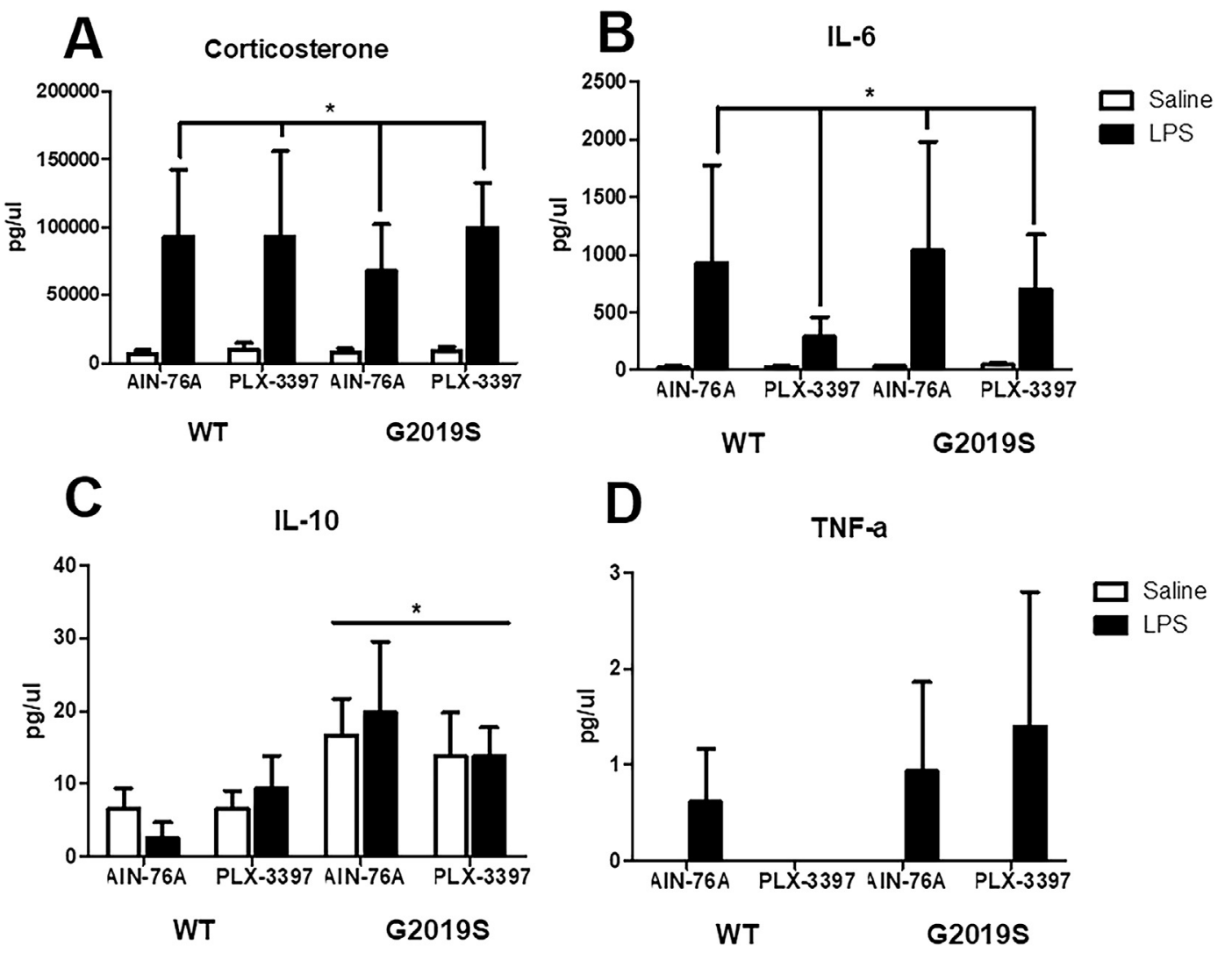

Fig. 5. Blood plasma levels of circulating factors were analyzed using ELISA and Luminex multiplex technology. The LPS and paraquat treatment resulted in increased levels of corticosterone (A) and IL-6 (B) and this was similarly evident in wild type (WT) and G2019S mutants. The G2019S KI increased levels of IL-10 (C), however, no statistically significant effects were found in TNF- $\alpha$ levels $(\mathrm{D} ; \mathrm{p}=0.09)$. In no case did the PLX-3397 pre-treatment induce any significant changes in cytokines. * $\mathrm{p}$ $<0.05$ compared to control animals.

levels following pre-treatment with PLX-3397 $(F(1,33)=31.940, p<$ 0.001) (Fig. 8A). Subsequent ELISAs run on serially extracted striatal samples found that the soluble alpha synuclein proteins levels were increased following PLX-3397 treatment, but no significant difference was observed for insoluble levels of the protein $(F(1,33)=16.136, p<$ 0.001 Fig. 8B,C).

\section{Discussion}

PD results from the highly complex interaction between environmental insults and underlying genetic vulnerabilities. Despite late symptom onset, there is strong evidence supporting the idea that the pathophysiology of PD may develop over a number of years with a long prodromal phase (Poewe et al., 2017; Noyce et al., 2016), which of course could provide time for intervention when appropriate mechanisms are targeted. While the precise pathophysiology of PD has not yet been fully elucidated, it is now commonly accepted that immune cells (e.g. microglia) play a very important role in promoting the degeneration of SNc dopamine neurons (Suzumura et al., 2006; Mosley et al., 2012; Machado et al., 2016). Microglia are a unique cell derived from primitive yolk-sac macrophages and engage both neuroprotective and inflammatory processes to maintain homeostasis (Ginhoux et al., 2013). During their normal surveillance state, these cells have highly ramified processes that constantly monitor their environment and upon activation undergo structural changes that allow for responses to the potential insult (Neumann et al., 2008). However, it has been posited that in PD the homeostatic balance of microglial cells is shifted towards an inflammatory phenotype, which may contribute to the cell death of dopamine neurons (Lecours et al., 2018). Further, accumulating evidence implicates the LRRK2 gene, most likely in conjunction with environmental stressor exposure, as playing a role in shaping the inflammatory milieu present in PD (Kim et al., 2018; Russo et al., 2015a; Choi et al., 2015). Indeed, besides familial PD, the G2019S LRRK2 mutation may be a vulnerability factor for the idiopathic form of the disease. Moreover, LRRK2 was found to be present in high levels in peripheral lymphocytes and monocytes of PD patients (Cook et al., 2017b) and LRRK2 mutation provoked a peripheral innate interferon cytokine response that was related to enhanced brain inflammation in response to LPS (Kozina et al., 2018).

To assess the role of microglia and LRRK2 in PD we transiently depleted microglia via pharmacological inhibition of the colony stimulating factor-1 receptor, using PLX-3397, in the context of LPS plus paraquat exposure in G2019S LRRK2 mutants and wild type littermates. We found that microglial depletion at the time of intra-SNc LPS infusion did indeed block the sickness, weight loss and mortality, which was in fact, most prominent in the LRRK2 G2019S mutant mice. Likewise, the reduced home-cage activity that was apparent following the paraquat regimen in the G2019S mice was also blunted in PLX-3397 treated mice. The PLX-3397 treatment was also associated with marked changes in several signaling factors within the SNc, including Sirt3 and caspases 1 and 3, as well as CX3CR1 and it also increased soluble levels of a-synuclein. However, the PLX-3397 pre-treatment did not influence the loss of SNc dopamine neurons that was provoked by LPS and paraquat administration. Similarly, the G2019S knock-in mutation also did not influence neuronal loss. The G2019S mutation did however influence microglial morphological state and interestingly, this was blocked by the previous PLX-3397 treatment.

This work was primarily guided by two hypotheses: 1 . Depleting microglia at the time of initiation of a dual hit LPS-paraquat regimen would prevent PD-like pathology and conversely, 2. That the G2019S knock-in mutation would augment PD-like pathology. It is important to 

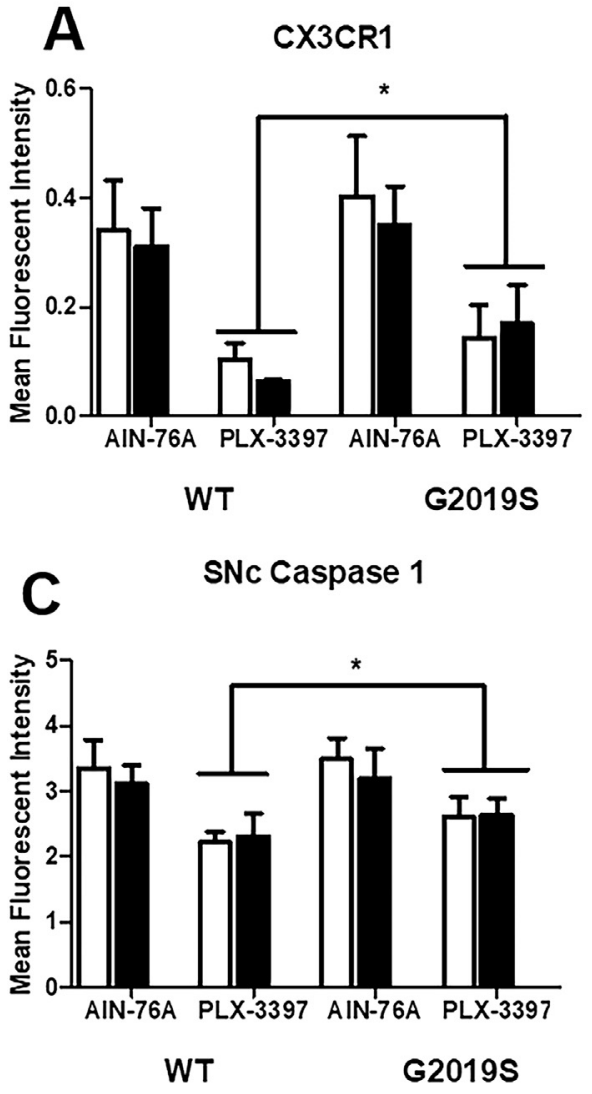

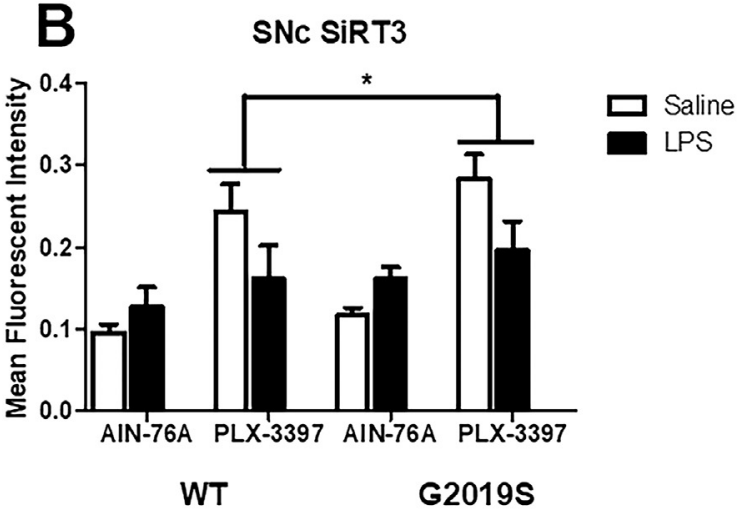

SNc Caspase 3

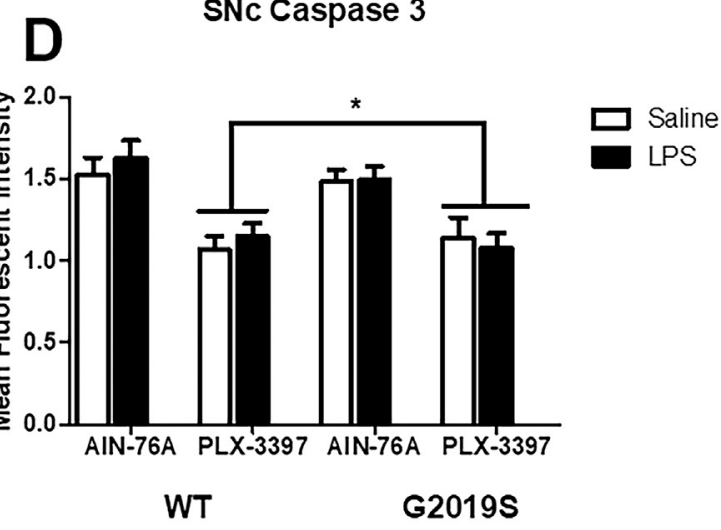

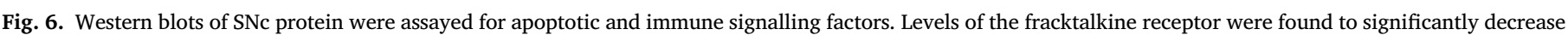

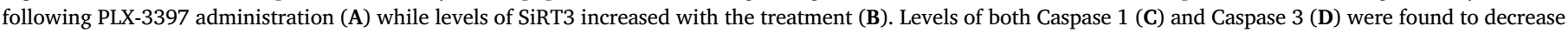
following PLX-3397 treatment. In no instance, did the LPS + paraquat or genotype variables affect these outcomes. ${ }^{*} \mathrm{p}<0.05$ compared to control animals.

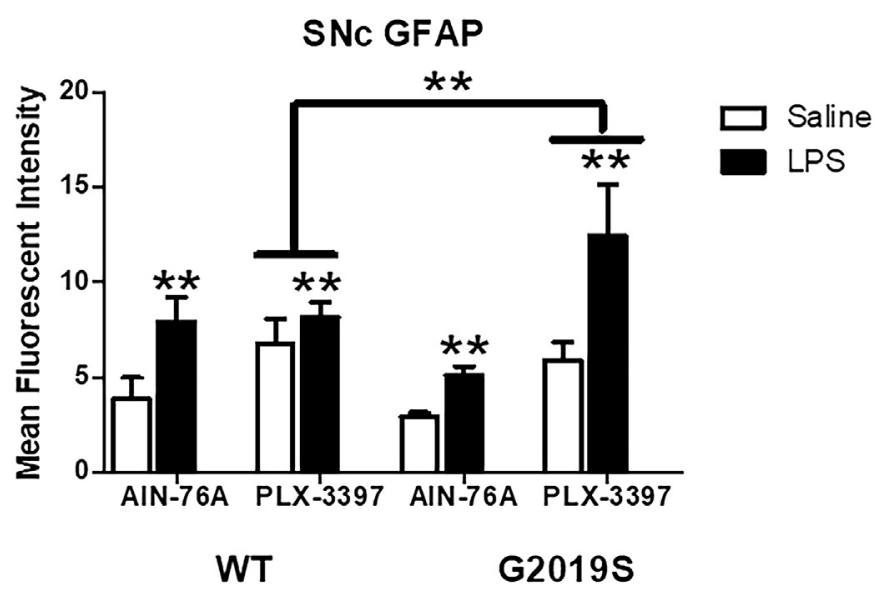

Fig. 7. Western blots probing for expression of the astrocytic marker, GFAP, revealed significantly increased levels within the SNc following LPS and paraquat administration in both wild type (WT) and G2019S mutants. Interestingly however, this marker was also increased by PLX-3397 administration, but this effect was confined to the G2019S mutant mice **p $<0.01$ compared to WT control animals.

underscore that microglia are only transiently depleted at the start of the experimental treatments, such that at the time of LPS infusion there should be $<10 \%$ remaining and hence, few TLR4 bearing cells for LPS to actually stimulate. During the subsequent week and a half of paraquat exposure, the microglia will be expected to be re-populating. Based on previous studies re-population beings as early as 3 days and is complete sometime between 7-14 days (Elmore et al., 2014), suggesting that the repopulation process should be almost complete by the time of sacrifice. This allows for evaluation of the very novel questions as to whether the neurodegenerative process is altered in the face of a depleted microglial population that is undergoing renewal. One study that was recently published using MPTP found that PLX-3397 treatment prior to (for 3 weeks) and during the entire toxicant injection period (7 days) actually resulted in augmented neurotoxicity with an increased loss of SNc dopamine neurons (Yang et al., 2018). Interestingly, increased infiltration of circulating leukocytes and pro-inflammatory cytokines were also observed with the four weeks of PLX-3397 treatment (and MPTP treatment) (Yang et al., 2018). This suggests that a peripheral inflammatory signal may have been recruited in the face of microglial depletion, perhaps through astrocytic intervention. Yet, it is important to underscore that microglia repopulation itself following PLX-3397 appears to stem from local microglial progenitor cells and not infiltrating peripheral bone marrow derived monocytes (Elmore et al., 2014, 2018bib_Elmore_et_al_2014bib_Elmore_et_al_2018). It is also important to note that PLX-3397 does not appreciably deplete peripheral macrophages or other immune cells, but is relatively selective for microglia (Mok et al., 2014). This contrasts with older viral based studies that used intracerebroventricular ganciclovir treatment in CD11b-HSVTK mice, which concluded that microglia were re-populated from peripheral monocytes (Varvel et al., 2012).

The few recent studies that have assessed microglial repopulation following PLX-3397 have remarkably found that re-populating microglia are largely normal in physiology and functional phenotype (Elmore et al., 2014). That said, there are obvious morphological differences (including larger cell bodies) at intermediate stages before the re-population is complete (Elmore et al., 2014). Further, cortical microglia were found to display reduced branching complexity of processes at 14 days but return to normal by 21 days. Yet, microglia appear to be less capable of 

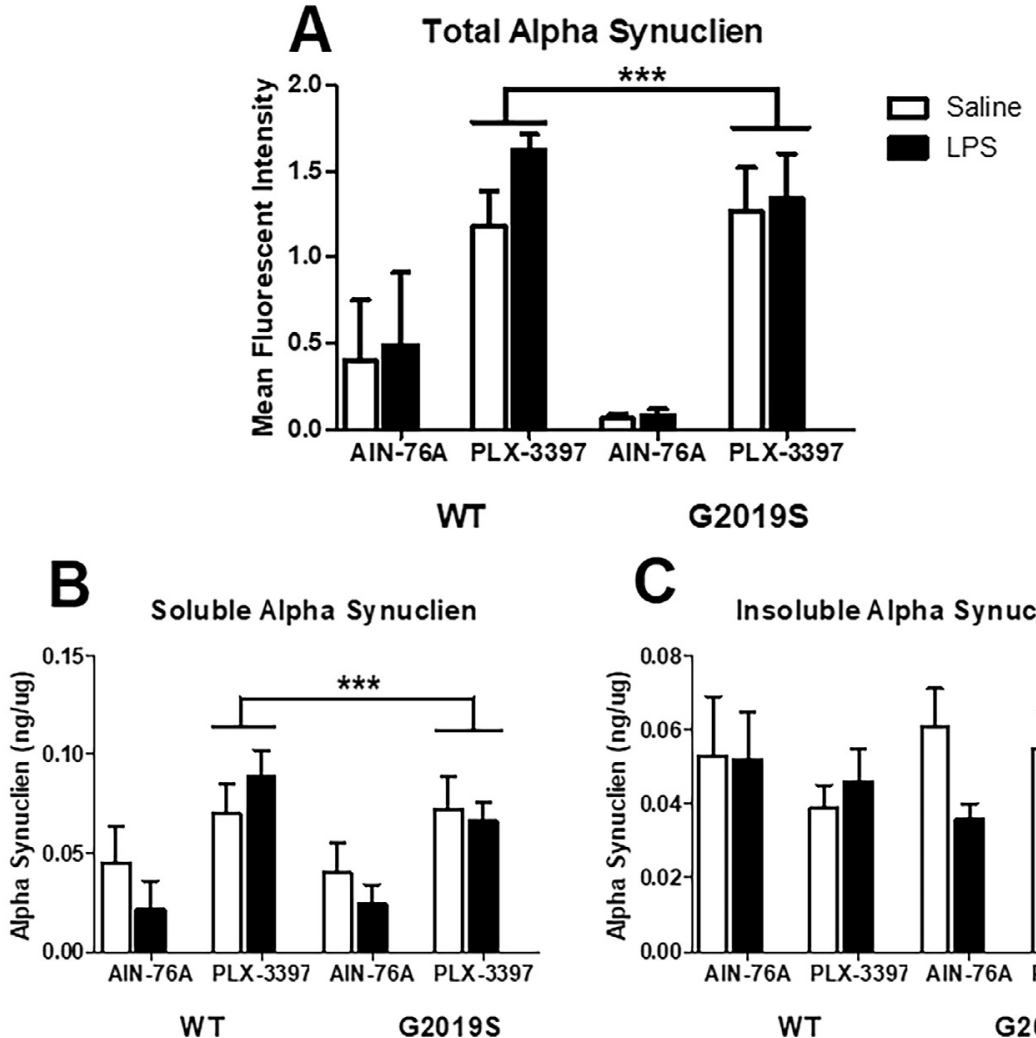

re-population following repeated episode of depletion (Najafi et al., 2018). Hence, although these are extremely plastic cells, there do appear to be limits to their long-term resilience. Further consideration should be given to the fact that microglial re-population may also vary as a function of brain region as differences in re-population rate and morphology were reported with the hippocampus and cortex (Elmore et al., 2014). For instance, while microglial re-population was complete in hippocampus after 14 days, it took 21 days to be fully restored in the prefrontal cortex (Elmore et al., 2014). Furthermore, the morphology of re-populated microglia appeared to vary between brain regions, with the average diameter of microglial processes increased in the hippocampus but not the cortex (Elmore et al., 2014).

While the mediators of microglial reactivity to immune priming are not clear, we hypothesize that LRRK2 plays a critical role favoring an enhanced inflammatory state. Indeed, among the alleles implicated in $\mathrm{PD}$, polymorphisms in the gene LRRK2 are the most common and its incomplete penetrance $(\sim 30-70 \%)$ suggests a role for environmental and/or immune factors as "triggers" (Hulihan et al., 2008; Lee et al., 2017b). While rapid-acting toxin-based models of PD such as MPTP and 6-OHDA have been extensively utilized in the context of LRRK2, relatively little work has yet examined more etiologically relevant models such as paraquat which act over time and with a less virulent lesion but more comprehensive range of PD-like pathology. Moreover, LRRK2 mutations were previously reported to enhance microglial reactivity and pro-inflammatory cytokine production in the brain and periphery (Russo et al., 2015b; Ho et al., 2018; Ma et al., 2016). In agreement with these findings, we did presently observe that G2019S knock-in mice had microglia morphology consistent with an "activated" state and this was evident even in the absence of the toxin treatments. Curiously, the G2019S mice also had basally elevated circulating IL-10 levels, but not that of pro-inflammatory cytokines (IL-1b, IL-6 or TNF-a). Although this finding seems paradoxical, it is consistent with a recent clinical trial that also showed elevated serum IL-10 levels in PD patients (Brockmann et al., 2016). Of course, timing of sacrifice could influence the nature of
Fig. 8. Total alpha Synuclein levels were quantified in the ipsilateral striatum via western blotting and were found to significantly increase with PLX-3397 treatment but was unaffected by genotype or LPS + paraquat treatment (A). The same region was also extracted with a detergent free and SDS containing buffer in order to assess soluble and insoluble levels of alpha-synuclein using an ELISA. It was found that soluble alpha synuclein increased in PLX-3397 treated animals (B), whereas insoluble did not (C). Again, there was no significant effect of genotype of LPS and paraquat treatment. $* * * \mathrm{p}$ $<0.001$ compared to AIN-76A control animals. 
treatments. It is also possible that the G2019S mutation may have affected the microglial phenotype by modulating its re-population lifecycle. Indeed, the depletion and re-population processes itself could be important since it was in fact reported that re-populating microglia initially expressed signs of an "activated" phenotype. Specifically, these microglia had increased expression of 196 genes, with many being proinflammatory cytokines (e.g. IFN-g), signaling factors (e.g. JAK-STAT) and pathogen detection factors (e.g. TLRs) (Elmore et al., 2018; Najafi et al., 2018).

\section{Conclusion}

In summary, we found that G2019S augments behavioral but not the neurodegenerative effects of LPS and paraquat treatment and similarly, that microglial depletion prior to toxin exposure blunts the behavioral toxicity but not the loss of dopaminergic neurons. Both the G2019S mutation and initial microglial depletion elicited complex effects upon microglial morphology, along with numerous changes in the SNc inflammatory and pro-death factors. Also, of particular note was that microglial depletion elevated levels of soluble a-synuclein in the striatum, which could reflect deficiencies in phagocytic clearance mechanisms obviously stemming from a loss of microglia and supported by our finding reduced CX3CR1 levels. Finally, elevated circulating levels of the stress hormone, corticosterone, and inflammatory cytokine, IL-6, is consistent with general distress induced by LPS and paraquat but this was unaffected by PLX-3397 treatment or G201S mutation. Yet, the G2019S mutants had elevated basal IL-10 levels. These data collectively support the contention that microglia are fundamentally critical for sickness and behavioral changes in response to inflammatory-toxin exposure, but not for the neurodegenerative response. It is also clear that the G2019S mutation has complex effects on the inflammatory milieu but is not necessarily a causative factor in PD-like pathology in the face of environmental stressors.

\section{Contributions}

This works contains significant contributions from each author. ZD, $\mathrm{CR}$, SH conceived and designed the experiments: Performed the experiments: CR, ZD. Contributed reagents/materials/analysis tools: SH. Analyzed the data and wrote the paper: $\mathrm{CR}, \mathrm{ZD}, \mathrm{SH}$.

\section{Availability of data and materials}

All data supporting the conclusions of this article will be included with this article.

\section{Ethics approval and consent to participate}

The Carleton University Committee for Animal Care approved all experimental procedures and complied with the guidelines set out by the Canadian Council for the Use and Care of Animals in Research.

\section{Consent for publication}

Not applicable.

\section{Declaration of competing interest}

The authors declare that they have no competing interests.

\section{Acknowledgements}

This work was supported by grants from the Canadian Institutes of Health Research (CIHR) and Natural Sciences and Engineering Research Council (NSERC) to S.H. CLINT membership is comprised of E. Schurr, Earl Brown, Derrick Gibbings, Shawn Hayley, David Park, Dana C.
Philpott, John D. Rioux, and Michael Schlossmacher.

\section{Abbreviations}

$\begin{array}{ll}\text { LPS } & \text { lipopolysaccharide } \\ \text { LRRK2 } & \text { Leucine rich repeat kinase-2 } \\ \text { MMx } & \text { Micromax } \\ \text { PD } & \text { Parkinson's disease } \\ \text { WT } & \text { Wild type KI: Knock-in }\end{array}$

\section{References}

Arimoto, T., Choi, D.-Y., Lu, X., Liu, M., Nguyen, X.V., Zheng, N., et al., 2007. Interleukin10 protects against inflammation-mediated degeneration of dopaminergic neurons in substantia nigra. Neurobiol. Aging 28, 894-906. https://doi.org/10.1016/ j.neurobiolaging.2006.04.011.

Ascherio, A., Schwarzschild, M.A., 2016. The epidemiology of Parkinson's disease: risk factors and prevention. Lancet Neurol. 15, 1257-1272. https://doi.org/10.1016/ S1474-4422(16)30230-7.

Baltazar, M.T., Dinis-Oliveira, R.J., de Lourdes Bastos, M., Tsatsakis, A.M., Duarte, J.A., Carvalho, F., 2014. Pesticides exposure as etiological factors of Parkinson's disease and other neurodegenerative diseases-A mechanistic approach. Toxicol. Lett. 230, 85-103.

Beier, E.E., Neal, M., Alam, G., Edler, M., Wu, L.-J., Richardson, J.R., 2017. Alternative microglial activation is associated with cessation of progressive dopamine neuron loss in mice systemically administered lipopolysaccharide. Neurobiol. Dis. 108, 115-127. https://doi.org/10.1016/j.nbd.2017.08.009.

Bobyn, J., Mangano, E.N., Gandhi, A., Nelson, E., Moloney, K., Clarke, M., et al., 2012. Viral-toxin interactions and Parkinson's disease: poly I:C priming enhanced the neurodegenerative effects of paraquat. J. Neuroinflammation 9, 86. https://doi.org/ 10.1186/1742-2094-9-86.

Brockmann, K., Apel, A., Schulte, C., Schneiderhan-Marra, N., Pont-Sunyer, C., Vilas, D., et al., 2016. Inflammatory profile in LRRK2-associated prodromal and clinical PD. J. Neuroinflammation 13, 122. https://doi.org/10.1186/s12974-016-0588-5.

Choi, I., Kim, B., Byun, J.-W., Baik, S.H., Huh, Y.H., Kim, J.-H., et al., 2015. LRRK2 G2019S mutation attenuates microglial motility by inhibiting focal adhesion kinase. Nat. Commun. 6, 8255. https://doi.org/10.1038/ncomms9255.

Cook, D.A., Kannarkat, G.T., Cintron, A.F., Butkovich, L.M., Fraser, K.B., Chang, J., et al., 2017. LRRK2 levels in immune cells are increased in Parkinson's disease. npj Park Dis. 3,11 .

Cook, D.A., Kannarkat, G.T., Cintron, A.F., Butkovich, L.M., Fraser, K.B., Chang, J., et al., 2017. LRRK2 levels in immune cells are increased in Parkinson's disease. npj Park Dis. 3, 11. https://doi.org/10.1038/s41531-017-0010-8.

Dick, F.D., De Palma, G., Ahmadi, A., Scott, N.W., Prescott, G.J., Bennett, J., et al., 2007. Environmental risk factors for Parkinson's disease and parkinsonism: the Geoparkinson study. Occup. Environ. Med. 64, 666-672. https://doi.org/10.1136/ oem.2006.027003.

Elmore, M.R.P., Najafi, A.R., Koike, M.A., Dagher, N.N., Spangenberg, E.E., Rice, R.A., et al., 2014. Colony-stimulating factor 1 receptor signaling is necessary for microglia viability, unmasking a microglia progenitor cell in the adult brain. Neuron 82 , 380-397. https://doi.org/10.1016/j.neuron.2014.02.040.

Elmore, M.R.P., Hohsfield, L.A., Kramár, E.A., Soreq, L., Lee, R.J., Pham, S.T., et al., 2018. Replacement of microglia in the aged brain reverses cognitive, synaptic, and neuronal deficits in mice. Aging Cell 17, e12832. https://doi.org/10.1111/acel.12832.

German, D.C., Eagar, T., Sonsalla, P.K., 2011. Parkinson's disease: a role for the immune system. Curr. Mol. Pharmacol. http://www.ncbi.nlm.nih.gov/pubmed/21675953 Accessed 11 Jun 2019.

Ginhoux, F., Lim, S., Hoeffel, G., Low, D., Huber, T., 2013. Origin and differentiation of microglia. Front. Cell. Neurosci. 7, 45. https://doi.org/10.3389/fncel.2013.00045.

Hakimi, M., Selvanantham, T., Swinton, E., Padmore, R.F., Tong, Y., Kabbach, G., et al., 2011. Parkinson's disease-linked LRRK2 is expressed in circulating and tissue immune cells and upregulated following recognition of microbial structures. J. Neural. Transm. 118.

Herzig, M.C., Kolly, C., Persohn, E., Theil, D., Schweizer, T., Hafner, T., et al., 2011. LRRK2 protein levels are determined by kinase function and are crucial for kidney and lung homeostasis in mice. Hum. Mol. Genet. 20, 4209-4223. https://doi.org/ 10.1093/hmg/ddr348.

Ho, D.H., Kim, H., Nam, D., Sim, H., Kim, J., Kim, H.G., et al., 2018. LRRK2 impairs autophagy by mediating phosphorylation of leucyl-tRNA synthetase. Cell Biochem. Funct. 36, 431-442. https://doi.org/10.1002/cbf.3364.

Hulihan, M.M., Ishihara-Paul, L., Kachergus, J., Warren, L., Amouri, R., Elango, R., et al., 2008. LRRK2 Gly2019Ser penetrance in Arab-Berber patients from Tunisia: a casecontrol genetic study. Lancet Neurol. 7, 591-594. https://doi.org/10.1016/S14744422(08)70116-9.

Johnston, L.C., Su, X., Maguire-Zeiss, K., Horovitz, K., Ankoudinova, I., Guschin, D., et al., 2008. Human interleukin-10 gene transfer is protective in a rat model of Parkinson's disease. Mol. Ther. 16, 1392-1399. https://doi.org/10.1038/MT.2008.113.

Kim, K.S., Marcogliese, P.C., Yang, J., Callaghan, S.M., Resende, V., Abdel-Messih, E., et al., 2018. Regulation of myeloid cell phagocytosis by LRRK2 via WAVE2 complex stabilization is altered in Parkinson's disease. Proc. Natl. Acad. Sci. Unit. States Am. 115, E5164-E5173. https://doi.org/10.1073/pnas.1718946115. 
Kozina, E., Sadasivan, S., Jiao, Y., Dou, Y., Ma, Z., Tan, H., et al., 2018. Mutant LRRK2 mediates peripheral and central immune responses leading to neurodegeneration in vivo. Brain 141 (6), 1753-1769.

Kuss, M., Adamopoulou, E., Kahle, P.J., 2014. Interferon- $\gamma$ induces leucine-rich repeat kinase LRRK2 via extracellular signal-regulated kinase ERK5 in macrophages. J. Neurochem. 129, 980-987. https://doi.org/10.1111/jnc.12668.

Lecours, C., Bordeleau, M., Cantin, L., Parent, M., Paolo, T Di, Tremblay, M.-È., 2018. Microglial implication in Parkinson's disease: loss of beneficial physiological roles or gain of inflammatory functions? Front. Cell. Neurosci. 12, 282. https://doi.org/ 10.3389/fncel.2018.00282.

Lee, A.J., Wang, Y., Alcalay, R.N., Mejia-Santana, H., Saunders-Pullman, R., Bressman, S., et al., 2017. Penetrance estimate of $L R R K 2$ p.G2019S mutation in individuals of nonAshkenazi Jewish ancestry. Mov. Disord. 32, 1432-1438. https://doi.org/10.1002/ mds. 27059.

Lee, A.J., Wang, Y., Alcalay, R.N., Mejia-Santana, H., Saunders-Pullman, R., Bressman, S., et al., 2017. Penetrance estimate of LRRK2 p.G2019S mutation in individuals of nonAshkenazi Jewish ancestry. Mov. Disord. 32, 1432-1438. https://doi.org/10.1002/ mds.27059.

Litteljohn, D., Mangano, E., Clarke, M., Bobyn, J., Moloney, K., Hayley, S., 2010. Inflammatory mechanisms of neurodegeneration in toxin-based models of Parkinson's disease. Parkinsons Dis. 2011, 713517.

Litteljohn, D., Nelson, E., Hayley, S., 2014. IFN-gamma differentially modulates memoryrelated processes under basal and chronic stressor conditions. Front. Cell. Neurosci. 8, 391.

Liu, Z., Bryant, N., Kumaran, R., Beilina, A., Abeliovich, A., Cookson, M.R., et al., 2018. LRRK2 phosphorylates membrane-bound Rabs and is activated by GTP-bound Rab7L1 to promote recruitment to the trans-Golgi network. Hum. Mol. Genet. 27, 385-395. https://doi.org/10.1093/hmg/ddx410.

Ma, B., Xu, L., Pan, X., Sun, L., Ding, J., Xie, C., et al., 2016. LRRK2 modulates microglial activity through regulation of chemokine (C-X3-C) receptor 1 -mediated signalling pathways. Hum. Mol. Genet. 25, 3515-3523. https://doi.org/10.1093/hmg/ ddw194.

Machado, V., Zöller, T., Attaai, A., Spittau, B., 2016. Microglia-mediated neuroinflammation and neurotrophic factor-induced protection in the MPTP mouse model of Parkinson's disease-lessons from transgenic mice. Int. J. Mol. Sci. 17.

Mangano, E.N., Hayley, S., 2009. Inflammatory priming of the substantia nigra influences the impact of later paraquat exposure: neuroimmune sensitization of neurodegeneration. Neurobiol. Aging 30, 1361-1378. https://doi.org/10.1016/ j.neurobiolaging.2007.11.020.

Mangano, E.N., Peters, S., Litteljohn, D., So, R., Bethune, C., Bobyn, J., et al., 2011. Granulocyte macrophage-colony stimulating factor protects against substantia nigra dopaminergic cell loss in an environmental toxin model of Parkinson's disease. Neurobiol. Dis. 43, 99-112.

Mangano, E.N., Litteljohn, D., So, R., Nelson, E., Peters, S., Bethune, C., et al., 2012. Interferon- $\gamma$ plays a role in paraquat-induced neurodegeneration involving oxidative and proinflammatory pathways. Neurobiol. Aging 33, 1411-1426.

Marder, K., Wang, Y., Alcalay, R.N., Mejia-Santana, H., Tang, M.-X., Lee, A., et al., 2015. Age-specific penetrance of LRRK2 G2019S in the Michael J. Fox ashkenazi jewish LRRK2 consortium. Neurology 85, 89-95. https://doi.org/10.1212/ WNL.0000000000001708.

Moehle, M.S., Daher, J.P.L., Hull, T.D., Boddu, R., Abdelmotilib, H.A., Mobley, J., et al., 2015. The G2019S LRRK2 mutation increases myeloid cell chemotactic responses and enhances LRRK2 binding to actin-regulatory proteins. Hum. Mol. Genet. 24, 4250-4267. https://doi.org/10.1093/hmg/ddv157.

Mok, S., Koya, R.C., Tsui, C., Xu, J., Robert, L., Wu, L., et al., 2014. Inhibition of CSF-1 receptor improves the antitumor efficacy of adoptive cell transfer immunotherapy. Canc. Res. 74, 153-161. https://doi.org/10.1158/0008-5472.CAN-13-1816.

Mosley, R.L., Hutter-Saunders, J.A., Stone, D.K., Gendelman, H.E., 2012. Inflammation and adaptive immunity in Parkinson's disease. Cold Spring Harb. Perspect Med. 2, a009381. https://doi.org/10.1101/cshperspect.a009381.
Mount, M.P., Lira, A., Grimes, D., Smith, P.D., Faucher, S., Slack, R., et al., 2007. Involvement of interferon- in microglial-mediated loss of dopaminergic neurons. J. Neurosci. 27, 3328-3337. https://doi.org/10.1523/JNEUROSCI.5321-06.2007.

Najafi, A.R., Crapser, J., Jiang, S., Ng, W., Mortazavi, A., West, B.L., et al., 2018. A limited capacity for microglial repopulation in the adult brain. Glia 66, 2385-2396. https:// doi.org/10.1002/glia.23477.

Neumann, H., Kotter, M.R., Franklin, R.J.M., 2008. Debris clearance by microglia: an essential link between degeneration and regeneration. Brain 132, 288-295. https:// doi.org/10.1093/brain/awn109.

Neumann, H., Kotter, M.R., Franklin, R.J.M., 2009. Debris clearance by microglia: an essential link between degeneration and regeneration. Brain 132, 288-295.

Noyce, A.J., Lees, A.J., Schrag, A.-E., 2016. The prediagnostic phase of Parkinson's disease. J. Neurol. Neurosurg. Psychiatry 87, 871-878. https://doi.org/10.1136/ jnnp-2015-311890.

Poewe, W., Seppi, K., Tanner, C.M., Halliday, G.M., Brundin, P., Volkmann, J., et al., 2017. Parkinson disease. Nat. Rev. Dis. Prim. 3, 17013. https://doi.org/10.1038/ nrdp.2017.13.

Purisai, M.G., McCormack, A.L., Cumine, S., Li, J., Isla, M.Z., Di Monte, D.A., 2007. Microglial activation as a priming event leading to paraquat-induced dopaminergic cell degeneration. Neurobiol. Dis. 25, 392-400. https://doi.org/10.1016/ j.nbd.2006.10.008.

Rudyk, C., Dwyer, Z., Hayley, S., membership, C.L.I.N.T., 2019. Leucine-rich repeat kinase-2 (LRRK2) modulates paraquat-induced inflammatory sickness and stress phenotype. J. Neuroinflammation 16, 120. https://doi.org/10.1186/s12974-0191483-7.

Russo, I., Berti, G., Plotegher, N., Bernardo, G., Filograna, R., Bubacco, L., et al., 2015. Leucine-rich repeat kinase 2 positively regulates inflammation and down-regulates NF-KB p50 signaling in cultured microglia cells. J. Neuroinflammation 12, 230.

Russo, I., Berti, G., Plotegher, N., Bernardo, G., Filograna, R., Bubacco, L., et al., 2015. Leucine-rich repeat kinase 2 positively regulates inflammation and down-regulates NF-кB p50 signaling in cultured microglia cells. J. Neuroinflammation 12, 230. https://doi.org/10.1186/s12974-015-0449-7.

Sawada, M., Imamura, K., Nagatsu, T., 2006. Role of cytokines in inflammatory process in Parkinson's disease. J. Neural. Transm. Suppl. 373-381.

Schildt, A., Walker, M.D., Dinelle, K., Miao, Q., Schulzer, M., O'Kusky, J., et al., 2019. Single inflammatory trigger leads to neuroinflammation in LRRK2 rodent model without degeneration of dopaminergic neurons. J. Parkinsons Dis. 9, 121-139. https://doi.org/10.3233/JPD-181446.

SUZUMURA, A., TAKEUCHI, H., ZHANG, G., KUNO, R., MIZUNO, T., 2006. Roles of gliaderived cytokines on neuronal degeneration and regeneration. Ann. N. Y. Acad. Sci. 1088, 219-229. https://doi.org/10.1196/annals.1366.012.

Tomlinson, J.J., Shutinoski, B., Dong, L., Meng, F., Elleithy, D., Lengacher, N.A., et al., 2017. Holocranohistochemistry enables the visualization of $\alpha$-synuclein expression in the murine olfactory system and discovery of its systemic anti-microbial effects. J. Neural. Transm. 124, 721-738. https://doi.org/10.1007/s00702-017-1726-7.

Uversky, V.N., Li, J., Fink, A.L., 2001. Pesticides directly accelerate the rate of alphasynuclein fibril formation : a possible factor in Parkinson's s disease. FEBS Lett. 500, 105-108.

Varvel, N.H., Grathwohl, S.A., Baumann, F., Liebig, C., Bosch, A., Brawek, B., et al., 2012. Microglial repopulation model reveals a robust homeostatic process for replacing CNS myeloid cells. Proc. Natl. Acad. Sci. U. S. A. 109, 18150-18155. https://doi.org/ 10.1073/pnas.1210150109.

Wu, W.Y.-Y., Kang, K.-H., Chen, S.L.-S., Chiu, S.Y.-H., Yen, A.M.-F., Fann, J.C.-Y., et al., 2015. Hepatitis C virus infection: a risk factor for Parkinson's disease. J. Viral Hepat. 22, 784-791. https://doi.org/10.1111/jvh.12392.

Yang, X., Ren, H., Wood, K., Li, M., Qiu, S., Shi, F.-D., et al., 2018. Depletion of microglia augments the dopaminergic neurotoxicity of MPTP. Faseb. J. 32, 3336-3345. https:// doi.org/10.1096/fj.201700833RR. 